Supporting Information

For

\title{
Exploiting An Imidazole-Functionalized Polyfluorene Derivative as A
}

\section{Chemosensory Material}

\author{
Xing-Hua Zhou, Ji-Chao Yan and Jian Pei*
}

The Key Laboratory of Bioorganic Chemistry and Molecular Engineering of Ministry of Education, College of Chemistry and Molecular Engineering, Peking University, Beijing 100871, China 


\section{Experimental Section}

General Methods. Chemicals were purchased from Aldrich, Acros and used as received.

${ }^{1} \mathrm{H}$ and ${ }^{13} \mathrm{C}$ NMR spectra were recorded on a Mercury plus $300 \mathrm{MHz}$ or Bruker $400 \mathrm{MHz}$ using $\mathrm{CDCl}_{3}$ as solvent in all cases. Elemental analyses were carried out on Elementar Vario EL (Germany). UV-vis spectra were recorded on PerkinElmer Lambda 35 UV-vis spectrometer. PL spectra were carried out on PerkinElmer LS 55 Luminescence Spectrometer.

We investigated the optical ion responsive properties of P1 in THF solutions through absorption and fluorescence spectra. The concentrations of $\mathbf{P 1}$ and of adding metal ions were $5 \times 10^{-6} \mathrm{M}$ and $5 \times 10^{-4} \mathrm{M}$, respectively. Control experiments showed that poly $(9,9-$ dihexylfluorene) did not exhibit any change in either absorption and emission spectra in the presence of any metal ions in THF solutions.

\section{2,7-Dibromo-9,9-bis(6'-bromohexyl)fluorene (1).}

To a mixture of 2,7-dibromofluorene $(9.72 \mathrm{~g}, 30.0 \mathrm{mmol})$ and catalyst amounts of tetrabutylammonium iodide in $\mathrm{DMSO}$ and $5 \mathrm{~mL}$ of $50 \%$ aqueous $\mathrm{NaOH}$ was added a solution of 1,6-dibromohexane $(24.4 \mathrm{~g}, 100.0 \mathrm{mmol})$ in DMSO. The reaction mixture was stirred for $24 \mathrm{~h}$ at the room temperature, and then poured into dilute hydrochloric acid. The aqueous layer was extracted by ethyl acetate. The combined extracts were washed with water, $\mathrm{NaHCO}_{3}$ solution, and brine and then dried over $\mathrm{MgSO}_{4}$. Removal of the solvent under vacuum followed by column chromatography using chloroform/petroleum ether produced a white solid (17.2 g, 88\%). ${ }^{1} \mathrm{H} \mathrm{NMR}\left(\mathrm{CDCl}_{3}, 300 \mathrm{MHz}, \mathrm{ppm}\right):$ 7.54-7.43 $(6 \mathrm{H}, \mathrm{m}, \mathrm{Ar}-\mathrm{H}), 3.32-3.27\left(4 \mathrm{H}, \mathrm{t}, J=6.9 \mathrm{~Hz}, \mathrm{CH}_{2}\right), 1.95-1.90\left(4 \mathrm{H}, \mathrm{m}, \mathrm{CH}_{2}\right), 1.72-1.62$ 
$\left(4 \mathrm{H}, \mathrm{m}, \mathrm{CH}_{2}\right), 1.25-1.03\left(8 \mathrm{H}, \mathrm{m}, \mathrm{CH}_{2}\right), 0.64-0.53\left(4 \mathrm{H}, \mathrm{m}, \mathrm{CH}_{2}\right) .{ }^{13} \mathrm{C}^{\mathrm{NMR}}\left(\mathrm{CDCl}_{3}, 75\right.$ MHz, ppm): 152.10, 139.00, 130.27, 126.02, 121.52, 121.19, 55.49, 40.00, 33.87, 32.56, 28.90, 27.71, 23.40. Anal. Calcd for $\mathrm{C}_{25} \mathrm{H}_{30} \mathrm{Br}_{4}$ : C, 46.19; H, 4.65; Br, 49.16. Found: C, 46.22; H, 4.98; Br, 49.06.

\section{2,7-Dibromo-9,9-bis(6'-(1-imidazole)hexyl)fluorene (2).}

A solution of imidazole $(0.75 \mathrm{~g}, 11.0 \mathrm{mmol})$ in anhydrous THF was added to a stirred suspension of $\mathrm{NaH}$ mineral oil $(0.26 \mathrm{~g}, 11.0 \mathrm{mmol})$ in $\mathrm{THF}$ at room temperature. The mixture was stirred for $1 \mathrm{~h}$ and then a solution of 2,7-dibromo-9,9-bis( $6^{\prime}$ bromohexyl)fluorine $(3.25 \mathrm{~g}, 5.0 \mathrm{mmol})$ and tetrabutylammonium iodide in THF was added. After $15 \mathrm{~h}$ at room temperature, the precipitate was filtered and washed with $\mathrm{CH}_{2} \mathrm{Cl}_{2}$. Evaporation of the solvent and purification by column chromatography (ethyl acetate) affored the product as a white solid $(2.03 \mathrm{~g}, 65 \%) .{ }^{1} \mathrm{H} \mathrm{NMR}\left(\mathrm{CDCl}_{3}, 300 \mathrm{MHz}\right.$, ppm): 7.54-7.51 (2H, d, $J=8.1 \mathrm{~Hz}, \mathrm{Ar}-\mathrm{H}), 7.48-7.45\left(2 \mathrm{H}, \mathrm{dd}, J_{1}=8.1 \mathrm{~Hz}, J_{2}=1.5 \mathrm{~Hz}\right.$, Ar-H), $7.41(2 \mathrm{H}, \mathrm{d}, J=1.5 \mathrm{~Hz}, \mathrm{Ar}-\mathrm{H}), 7.38(2 \mathrm{H}, \mathrm{s}, \mathrm{Ar}-\mathrm{H}), 7.02(2 \mathrm{H}, \mathrm{s}, \mathrm{Im}-\mathrm{H}), 6.82(2 \mathrm{H}$, s, Im-H), 3.82-3.77 (4H, t, $\left.J=7.2 \mathrm{~Hz}, \mathrm{CH}_{2}\right), 1.91-1.86\left(4 \mathrm{H}, \mathrm{m}, \mathrm{CH}_{2}\right), 1.59-1.55(4 \mathrm{H}, \mathrm{m}$, $\left.\mathrm{CH}_{2}\right), 1.07-1.04\left(8 \mathrm{H}, \mathrm{m}, \mathrm{CH}_{2}\right), 0.54\left(4 \mathrm{H}, \mathrm{m}, \mathrm{CH}_{2}\right) .{ }^{13} \mathrm{C} \mathrm{NMR}\left(\mathrm{CDCl}_{3}, 75 \mathrm{MHz}, \mathrm{ppm}\right)$ : $151.90,138.93,136.96,130.26,129.26,125.88,121.45,121.19,118.63,55.37,46.78$, 39.82, 30.73, 29.04, 25.97, 23.23. Anal. Calcd for $\mathrm{C}_{31} \mathrm{H}_{36} \mathrm{Br}_{2} \mathrm{~N}_{4}$ : C, 59.63; H, 5.81; $\mathrm{Br}$, 25.59; N, 8.97. Found: C, 59.33; H, 5.83; Br, 25.32; N, 8.64.

\section{9,9-di-n-hexylfluorene-2,7-bis(trimethylene boronate) (3).}

In a three-necked flask 2,7-dibromo-9,9-di- $n$-hexyl-9H-fluorene (13.45 g, $27.3 \mathrm{mmol})$ was treated with magnesium (turnings) $(1.46 \mathrm{~g}, 60.1 \mathrm{mmol})$ in THF to form the Grinard reagent under nitrogen. The Grignard reagent solution was added slowly dropwise into a 
stirred solution of anhydrous trimethyl borate $(8.52 \mathrm{~g}, 82.0 \mathrm{mmol})$ in THF at $-78{ }^{\circ} \mathrm{C}$ under nitrogen. The mixture was then warmed to room temperature and stirred overnight, followed by concentrating to dryness by rotary evaporator distillation. To the resulting solid were added trimethylene glycol $(10 \mathrm{~mL})$ and anhydrous toluene $(150 \mathrm{~mL})$. The mixture was refluxed overnight, and then evaporated under vacuum. The residue was dissolved in chloroform, washed with $\mathrm{H}_{2} \mathrm{O}$, brine and dried over anhydrous magnesium sulfate. After removing the solvent, the crude product was purified by column chromatography using ethyl acetate/petroleum ether to afford $9.60 \mathrm{~g}(70 \%)$ of a white solid. Further purification could be achieved by recrystallization from petroleum ether. ${ }^{1} \mathrm{H}$ NMR (CDCl $\left., 300 \mathrm{MHz}, \mathrm{ppm}\right):$ 7.74-7.70 (6H, m, Ar-H), 4.22-4.18 (8H, t, $J=5.4 \mathrm{~Hz}$, $\left.\mathrm{CH}_{2}\right), 2.08\left(4 \mathrm{H}, \mathrm{m}, \mathrm{CH}_{2}\right), 1.98\left(4 \mathrm{H}, \mathrm{m}, \mathrm{CH}_{2}\right), 1.20-0.90\left(12 \mathrm{H}, \mathrm{m}, \mathrm{CH}_{2}\right), 0.76-0.71(6 \mathrm{H}, \mathrm{t}$, $\left.J=7.2 \mathrm{~Hz}, \mathrm{CH}_{3}\right), 0.56\left(4 \mathrm{H}, \mathrm{m}, \mathrm{CH}_{2}\right) .{ }^{13} \mathrm{C} \mathrm{NMR}\left(\mathrm{CDCl}_{3}, 75 \mathrm{MHz}, \mathrm{ppm}\right): 151.17,140.03$, $139.18,132.44,128.10,123.53,119.83,109.94,55.09,40.28,31.53,31.43,30.82,29.62$, 29.04, 28.71, 23.76, 22.48, 13.94. Anal. Calcd for $\mathrm{C}_{31} \mathrm{H}_{44} \mathrm{~B}_{2} \mathrm{O}_{4}$ : C, 74.13; H, 8.83; Found: C, $74.03 ; \mathrm{H}, 8.66$.

\section{Poly[(9,9-di-n-hexyl-9H-fluoren-2,7-ylene)-co-alt-9,9-bis(6'-(1-imidazolene)hexyl)- 9H-fluoren-2,7-ylene] (P1).}

Under a nitrogen atmosphere, 9,9-dihexylfluorene-2,7-bis(trimethylene boronate) (0.753 $\mathrm{g}, 1.5 \mathrm{mmol}$ ) and 2,7-dibromo-9,9-bis(6'-(imidazole-1-ylene)hexyl)fluorene ( $0.937 \mathrm{~g}$, $1.5 \mathrm{mmol})$ were mixed together with $1.5 \mathrm{~mol} \%$ of $\mathrm{Pd}\left(\mathrm{PPh}_{3}\right)_{4}, 10 \mathrm{~mL}$ of degassed toluene and $6 \mathrm{~mL}$ of degassed $2 \mathrm{M}$ aqueous sodium carbonate solution. The mixture was vigorously stirred at $80-90{ }^{\circ} \mathrm{C}$ for $48 \mathrm{~h}$. The mixture was poured into stirred $100 \mathrm{~mL}$ of methanol to precipitate a plenty of solids. The solid was collected by filtration, and 
washed with methanol and water. The polymer was further purified by washing with refluxing acetone in Soxhlet for 2 days, and was dried under vacuum at room temperature to afford $0.73 \mathrm{~g}(61 \%)$ of a light yellow solid. ${ }^{1} \mathrm{H}$ NMR $\left(\mathrm{CDCl}_{3}, 300 \mathrm{MHz}, \mathrm{ppm}\right)$ : 7.887.85 (4H, m, Ar-H), 7.69-7.66 (8H, m, Ar-H), 7.36 (2H, s, Im-H), 7.00 (2H, s, Im-H), $6.78(2 \mathrm{H}, \mathrm{s}, \mathrm{Im}-\mathrm{H}), 3.80-3.76\left(4 \mathrm{H}, \mathrm{t}, J=6.9 \mathrm{~Hz}, \mathrm{CH}_{2}\right), 2.12\left(4 \mathrm{H}, \mathrm{m}, \mathrm{CH}_{2}\right), 1.76(4 \mathrm{H}, \mathrm{m}$, $\left.\mathrm{CH}_{2}\right), 1.59\left(4 \mathrm{H}, \mathrm{m}, \mathrm{CH}_{2}\right), 1.12\left(20 \mathrm{H}, \mathrm{m}, \mathrm{CH}_{2}\right), 0.79-0.77\left(14 \mathrm{H}, \mathrm{m}, \mathrm{CH}_{2}, \mathrm{CH}_{3}\right) .{ }^{13} \mathrm{C} \mathrm{NMR}$ $\left(\mathrm{CDCl}_{3}, 75 \mathrm{MHz}, \mathrm{ppm}\right): 151.81,151.29,140.54,140.30,140.09,136.94,129.27,127.17$, $126.35,121.36,120.12,118.65,55.36,55.22,46.86,40.33,31.42,30.87,29.61,29.41$, 26.19, 23.82, 22.50, 14.02. Anal. Calcd for $\mathrm{C}_{56} \mathrm{H}_{68} \mathrm{~N}_{4}:$ C, 84.37; H, 8.60; N, 7.03. Found: C, 84.10; H, 8.58; N, 6.90.

\section{2-(9,9-Di-n-hexylfluorenyl)[1,3,2] dioxaborolane (4).}

9,9-Dihexyl-2-fluorenylmagnesium bromide prepared from 2-bromo-9,9-di- $n$-hexylfluorene $(11.37 \mathrm{~g}, 27.5 \mathrm{mmol})$ and magnesium turning $(0.74 \mathrm{~g}, 30.4 \mathrm{mmol})$ in THF was added dropwise into a solution of trimethyl borate $(7.87 \mathrm{~g}, 75.7 \mathrm{mmol})$ in $\mathrm{THF}$ at $-78^{\circ} \mathrm{C}$. The reaction mixture was warmed to room temperature and stirred overnight, followed by concentrating to dryness by rotary evaporator distillation. To the resulting solid were added ethylene glycol $(15 \mathrm{~mL})$ and toluene $(100 \mathrm{~mL})$. The mixture was refluxed overnight, and the toluene layer was separated and concentrated under reduced pressure. The product was purified by column chromatography using ethyl acetate/petroleum ether to afford $8.35 \mathrm{~g}(75 \%)$ of a white solid. Further purification could be achieved by recrystallization from petroleum ether. ${ }^{1} \mathrm{H} \mathrm{NMR}\left(\mathrm{CDCl}_{3}, 300 \mathrm{MHz}, \mathrm{ppm}\right)$ : 7.82-7.78 $(2 \mathrm{H}$, m, Ar-H), 7.74-7.71 (2H, m, Ar-H), 7.35-7.30 (3H, m, Ar-H), $4.42\left(4 \mathrm{H}, \mathrm{s}, \mathrm{OCH}_{2}\right), 2.01-$ $1.94\left(4 \mathrm{H}, \mathrm{m}, \mathrm{CH}_{2}\right), 1.12-1.00\left(12 \mathrm{H}, \mathrm{m}, \mathrm{CH}_{2}\right), 0.77-0.72\left(6 \mathrm{H}, \mathrm{t}, J=6.9 \mathrm{~Hz}, \mathrm{CH}_{3}\right), 0.60-$ 
$0.56\left(4 \mathrm{H}, \mathrm{m}, \mathrm{CH}_{2}\right) .{ }^{13} \mathrm{C} \mathrm{NMR}\left(\mathrm{CDCl}_{3}, 75 \mathrm{MHz}, \mathrm{ppm}\right): 151.28,149.93,144.38,140.74$, $133.64,128.96,127.61,126.69,122.87,120.12,119.15,66.00,54.99,40.33,31.48$, 29.68, 23.68, 22.55, 13.96. Anal. Calcd for $\mathrm{C}_{27} \mathrm{H}_{37} \mathrm{BO}_{2}$ : C, 80.19; H, 9.22; B, 2.67; O, 7.91. Found: C, 80.23; H, 9.30. EI MS (m/e): $404\left(\mathrm{M}^{+}\right)$.

\section{9,9-bis(6'-(1-imidazolene)hexyl)-2,7-bis(9,9-di-n-hexylfluorene-2-yl)fluorene (5).}

To a solution of 2,7-Dibromo-9,9-bis(6'-(1-imidazole)hexyl)fluorene (1.25 g, $2.0 \mathrm{mmol})$ and 2-(9,9-dihexfluorenyl)[1,3,2]dioxaborolane $(2.43 \mathrm{~g}, 6.0 \mathrm{mmol})$ in toluene $(20 \mathrm{~mL})$ was added $\mathrm{Pd}\left(\mathrm{PPh}_{3}\right)_{4}(1.0 \mathrm{~mol} \%)$ and $2 \mathrm{M}$ aqueous $\mathrm{Na}_{2} \mathrm{CO}_{3}$ solution $(12 \mathrm{~mL})$. The mixture was degassed and stirred at $90{ }^{\circ} \mathrm{C}$ for $24 \mathrm{~h}$ and then was poured into a saturated solution of ammonium chloride and extracted with ethyl acetate, the combined organic layer was washed with brine and dried over $\mathrm{MgSO}_{4}$. After removing the solvent, the residue was purified by column chromatography using ethyl acetate as eluent to give 1.63 g (72\%) of a pale yellow solid. ${ }^{1} \mathrm{H}$ NMR $\left(\mathrm{CDCl}_{3}, 300 \mathrm{MHz}, \mathrm{ppm}\right)$ : 7.85-7.71 (6H, m, ArH), 7.67-7.63 (8H, m, Ar-H), 7.39-7.31 (8H, m, Ar-H), $6.97(2 \mathrm{H}, \mathrm{s}, \mathrm{Im}-\mathrm{H}), 6.75(2 \mathrm{H}, \mathrm{s}$, Im-H), 3.78-3.71 (4H, t, $\left.J=7.2 \mathrm{~Hz}, \mathrm{CH}_{2}\right), 2.08-2.00\left(12 \mathrm{H}, \mathrm{m}, \mathrm{CH}_{2}\right), 1.60-1.50(4 \mathrm{H}, \mathrm{m}$, $\left.\mathrm{CH}_{2}\right), 1.08\left(36 \mathrm{H}, \mathrm{m}, \mathrm{CH}_{2}\right), 0.80-0.73\left(20 \mathrm{H}, \mathrm{m}, \mathrm{CH}_{2}, \mathrm{CH}_{3}\right) .{ }^{13} \mathrm{C} \mathrm{NMR} \mathrm{CDCl}_{3}, 75 \mathrm{MHz}$, ppm): $151.45,151.15,150.80,140.56,140.45,140.41,140.05,139.91,136.85,129.18$, $127.02,126.75,126.23,125.88,122.84,121.14,121.07,120.05,119.87,119.68,118.57$, $55.11,55.08,46.77,40.30,31.38,30.84,29.60,29.35,26.12,23.68,22.48,13.98$. Anal. Calcd for $\mathrm{C}_{81} \mathrm{H}_{102} \mathrm{~N}_{4}$ : C, 85.97; H, 9.08; N, 4.95. Found: C, 85.74; H, 8.75; N, 4.77. 


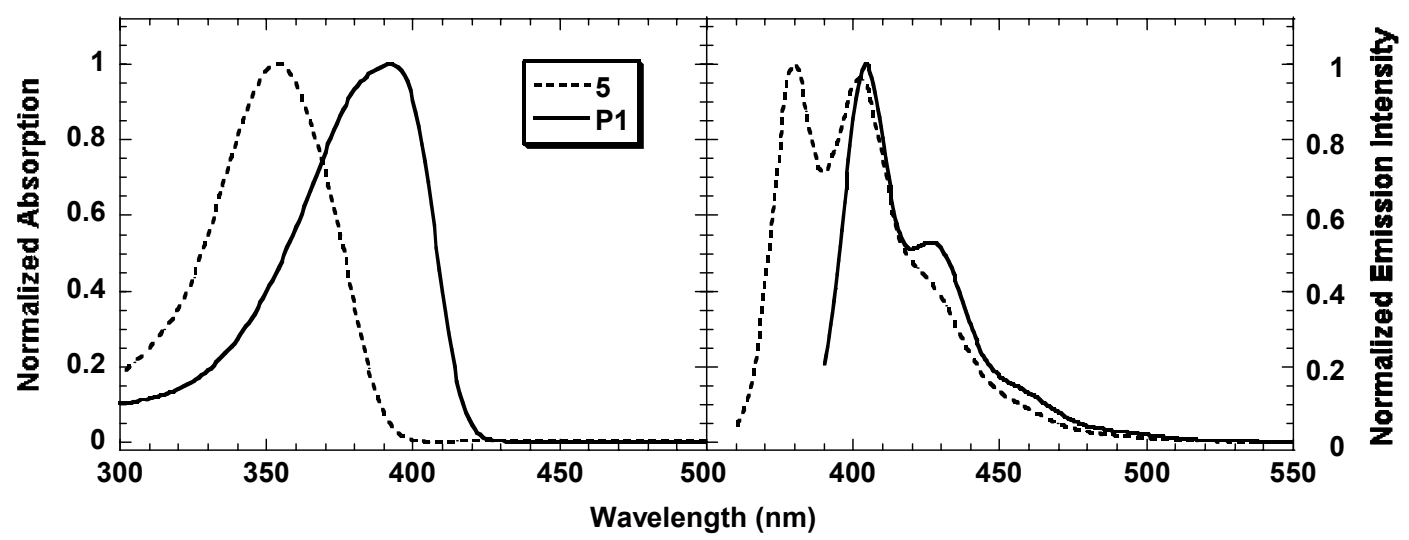

Figure S1. The UV-vis absorption and photoluminescence spectra of P1 (solid line) and its model $\mathbf{5}$ (dashed line) in THF solutions at room temperature.

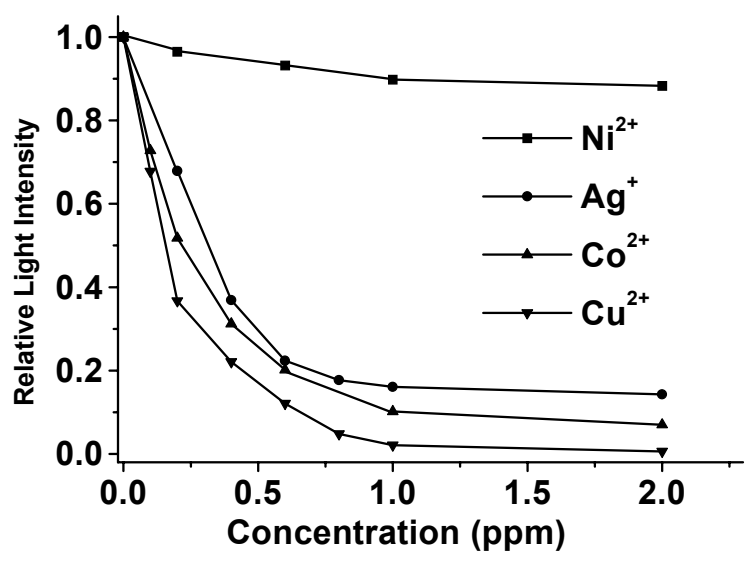

Figure S2. The titration curves for P1 upon addition of metal ions (from 0 ppm to 2 $\mathrm{ppm})$. The polymer concentration was $5 \times 10^{-6} \mathrm{M}$ corresponding to repeating unit. 


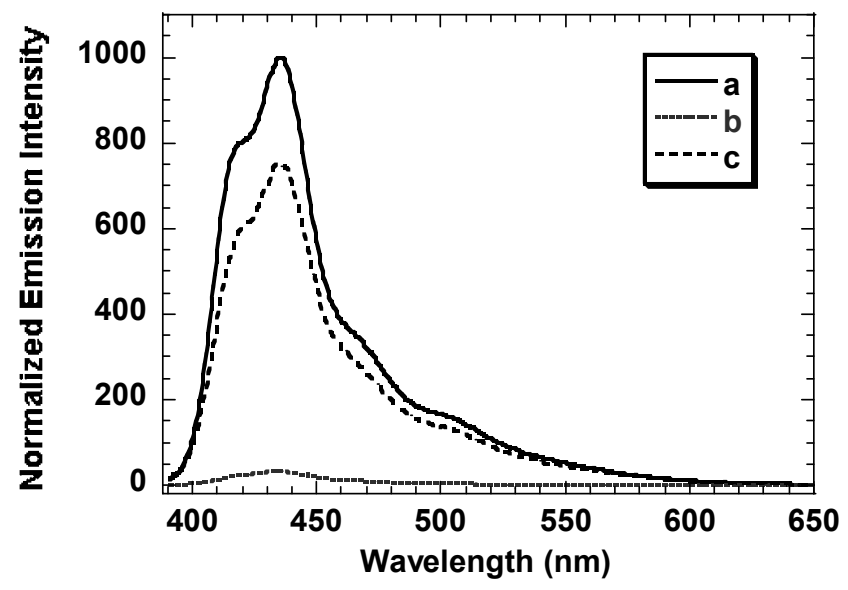

Figure S3. Fluorescence emission spectra P1 in solid states on a quartz plate: a) before immersion in $\mathrm{Cu}^{2+}$ aqueous solutions, b) after immersion in $\mathrm{Cu}^{2+}$ aqueous solutions, and c) after immersion in methanolic ammonia solutions. 